\title{
La valoración económica y mercantilización del agua de consumo humano en el Estado de México. Algunos determinantes
}

\section{Factors for economic assessment of water human consumption in the Estado de México. Some determinants}

doi: http://dx.doi.org/10.32870/

espiral.v24i68.4557

\section{Resumen}

El agua de consumo humano se ha convertido en uno de los recursos más complejos para asignarle un valor nominal, de ahí el objetivo de analizar algunos de los determinantes que influyen en la valoración económica y mercantilización de este recurso en el Estado de México. Este análisis está sustentado en algunos elementos de teoría económica que permiten realizar un contraste con las condiciones actuales de acceso y disfrute. Los resultados evidencian su carácter escaso y pecuniario, lo que amplía el riesgo y vulnerabilidad socioterritorial. Los determinantes propuestos amplían la visión transversal del agua en la ciencia económica y representan un hito que fortalece la discusión holística para generar propuestas concretas y viables.

Palabras clave: determinantes, agua de consumo humano, valor económico del agua, escasez y racionamiento del agua,

Estado de México.
David Iglesias Piña*

\begin{abstract}
Water for human consumption has become in one of the most complex and difficult resources of the market. The objective of this paper is to analyze some of the determinants influencing economic valuation and commercialization of this resource in the Estado de México. This analysis is supported by some elements of economic theory that allows contrast with the contemporary conditions of access and enjoyment. The results show the financial character, increasing the social risk and vulnerability. This paper extends the view of water in economic science. It's a milestone that strengthen the holistic discussion to generate concrete and viable proposals.
\end{abstract}

Keywords: determinants, drinking water, economic value of water, water shortages and rationing, Estado de México.

\footnotetext{
-Profesor-Investigador de la Universidad Autónoma del Estado de México.

diglesiasp@uaemex.mx

Fecha de recepción: 03 de diciembre de 2015. Fecha de aceptación: 03 de mayo de 2016.
} 


\section{Introducción}

La lucha y competencia por el acceso y disposición de los satisfactores básicos como el agua es más que evidente, al grado de hablarse de una crisis alimentaria mundial. El agua para consumo humano (potable) se ha convertido en un indicador importante para determinar el nivel de bienestar social y explicar su comportamiento contemporáneo en los diferentes entornos territoriales. Al cierre del año 2006, se estimaba que había en el mundo 1100 millones de personas que no tenían acceso al agua potable, y a finales de 2014 esta carestía creció a una tasa de $703.18 \%$, equivalente a 8835 millones de seres humanos sin dicho satisfactor.

En México, alrededor de doce millones de personas enfrentan este problema, en tanto que en el Estado de México 1.3 millones de habitantes todavía no disfrutan del vital recurso (FEA, et al., 2006; INEGI, 2010). En los medios urbanos, el problema es más latente, y se explica por la acelerada tasa de crecimiento demográfico, la transición productiva-sectorial, la modificación de los patrones de consumo, la alteración de los ciclos climáticos, la ubicación de las viviendas, las condiciones físico-geográficas del lugar, la traza territorial, la distribución del espacio, las condiciones de la infraestructura sanitaria, los costos de extracción y distribución, entre otros, que provocan que el acceso a dicho recurso sea cada vez más complejo.

Este cúmulo de factores, aunados al desaprovechamiento por fugas derivado del deterioro de la infraestructura y el costo monetario que ello implica para distribuir el recurso, reducen cada vez más las posibilidades de aumentar la cobertura, frecuencia y presión de disposición del agua potable, afectando la calidad de vida y los niveles de desarrollo social.

El objetivo de este documento es explicar y evidenciar los costos de disposición del agua para consumo humano 
en el Estado de México desde algunos posicionamientos de la teoría económica. Para ello, se resaltan algunos de los principales factores que influyen en una valoración nominal creciente, al grado de incluso concebir al agua como un recurso mercantilizado, provocando amplios contrastes sociales y territoriales, así como un panorama de carestía y conflicto humano mundial.

\section{La conversión nominal y mercantil del agua, algunos elementos fácticos}

Por su naturaleza, el agua es considerada un bien libre y público puro, ya que no es de acceso exclusivo, y, dada su cantidad ilimitada, no debe haber competencia para su consumo, exclusión en su uso ni mucho menos una disponibilidad racionada; más aún, su carácter libre permite que todos los individuos puedan disfrutar simultáneamente de dicho bien.

Es así que el agua pertenece al ambiente: desde las perspectivas preservacionista y del naturalismo, fundadas en las tesis geocéntricas (gaia) o biocéntricas (ecología profunda), se argumenta que no debe ser trastocada por las actividades del ser humano, pues este no posee ningún derecho sobre el agua y el resto de los recursos naturales, razón por la que estas mismas perspectivas se oponen directamente a la valoración económica del ambiente a través del mercado. Esto es, hay un rechazo de la economía de los recursos naturales y del ambiente (Corona, 2000).

A pesar de este precepto, el agua presenta una serie de características y atributos cuyo nivel de utilidad y grado de satisfacción socioambiental empieza no sólo a diferenciarse, sino a convertirla en un recurso altamente demandado y susceptible de apropiación. Este es el caso del agua dulce, indispensable para la sobrevivencia humana, razón por la que el hombre no sólo se ha apropiado su existencia, sino 
que se ha limitado su disposición y se ha valorado monetariamente su consumo, ya que el agua dulce no es tan abundante y su existencia ilimitada es sólo aparente, de ahí que su valoración mercantil y apropiación privada sean consideradas como reguladores de su explotación intensiva.

Cuando un recurso natural existe en abundancia no es un bien económico, sino un bien libre, por lo tanto no es objeto de valoración económica; más bien, para que un recurso natural o ambiental sea susceptible de asignársele un costo y precio en el mercado, debe cumplir una triple exigencia:

1) Tener existencia limitada.

2) Su consumo o disposición genera una utilidad vital y puede intercambiarse.

3) Puede ser industrialmente producible y multiplicable (obtención de una gama de productos con la utilización del agua) (Corona, 2000, p. 125).

Bajo esta consideración, y desde el punto de vista de la eficiencia económica, ahora el agua le pertenece al hombre para su consumo y para desarrollar un sinfín de actividades económicas y productivas, donde el desarrollo tecnológico y la existencia de sustitutos perfectos e imperfectos determinan el nivel de explotación de dicho recurso, todo en función de los precios, estando ausente toda consideración intra- e intergeneracional, lo que, por un lado, rompe con el principio de sostenibilidad, en términos del disfrute generacional de dicho recurso, mientras que por otro se acepta que la dinámica de los recursos naturales da lugar a una interpretación contemporánea de las relaciones entre economía y recursos naturales (coevolución ambiental). Por tanto, la centralidad universal que el agua tiene para la vida humana se incrementa en las cuencas, donde las restricciones naturales se unen a las de la intervención humana (Flores, 2014).

Desde la perspectiva del utilitarismo se otorga valor a todo aquello que contribuye positivamente al bienestar de 
la persona, razón del énfasis en el bienestar de los individuos como criterio de demarcación de los distintos valores. Vastos recursos ambientales como el agua dulce, que son necesarios para la sobrevivencia humana, tienen un costo que deriva de su utilidad, por lo que el proceso de valoración se basa en el reconocimiento de esta cualidad, y recientemente por su existencia en la naturaleza, de ahí que los seres humanos vayan otorgando o descubriendo valores funcionales, económicos, ecológicos, estéticos, culturales, históricos, ambientales, entre otros, no sólo del agua dulce, sino del conjunto de recursos que conforman el ambiente (Anderson, 1993; Azqueta, 2007).

El medio ambiente adquiere, pues, toda una serie de valores porque cumple una gama de funciones que afectan positivamente al bienestar, utilidad o deleite de las personas que componen la sociedad, bien sea como productor de bienes y servicios, espacio de placer, estímulo para la perfección personal, reducto de civilización o símbolo de cultura (Anderson, 1993, p. 79).

Es así que este recurso se convierte en un capital natural objeto de transacción o negociación entre las personas desde la perspectiva de la teoría económica clásica. El realismo ambiental asume que la idea más ingenua de los recursos de la naturaleza es que sean considerados como una entidad real (idealismo ambiental), siendo que el humano y la sociedad le han contrapuesto valores al propio esquema epistemológico para interpretar su utilidad individual y colectiva (Macnaghten y Urry, 1998).

De esta manera, la valoración económica del agua dulce gana terreno a medida que se expande la sociedad y el desarrollo de sus actividades económicas, por lo que su existencia enfrenta una serie de externalidades negativas irreversibles, siendo una de las más relevantes su escasez y contaminación, lo que ha obligado al Estado a instrumentar 
políticas públicas para una mejor extracción, distribución y aprovechamiento.

De esta manera, la política del agua se convierte en el fundamento de análisis de los problemas sociales y económicos, pero también la convierte en el recurso de mayor disputa entre los colectivos, ya que al ser uno de los elementos nodales para la generación de riqueza y bienestar social aumenta su atracción y competencia en el mercado. De hecho, la misma Conferencia sobre el Agua y el Medio Ambiente, también conocida como Declaración de Dublín, realizada en Irlanda en enero de 1992, señaló dentro de sus principios rectores que el agua dulce es un recurso finito y vulnerable, esencial para sostener la vida, el desarrollo y el medio ambiente, por lo que esta tiene un valor económico en todos sus diversos usos en competencia a los que se destina, y que debería reconocérsele como un bien económico (SEMARNAT-SER, 1992).

El desarrollo del mercado global para el agua está emergiendo porque la consideración se está moviendo rápidamente desde el concepto de gestión pública del recurso hacia la atención al agua como bien económico, ya que las agencias públicas se han mostrado ineficientes a la hora de garantizar su disponibilidad como necesidad básica, en cantidad y calidad suficiente y bajo una adecuada gestión para todos los seres humanos (Gleick, et al., 2002, III).

El argumento de que ahora el agua para consumo humano sea considerada como recurso económico estriba en que sólo el $0.007 \%$ (equivalente a $4200 \mathrm{~km}^{3}$ ) del agua disponible en el planeta es dulce y apta para consumo directo de los seres humanos. Si esta cantidad se divide entre los aproximadamente 6000 millones de habitantes del mundo, se tiene que cada persona dispone de $700 \mathrm{~m}^{3}$ anualmente para su sobrevivencia. Sin embargo, esta cantidad no refleja la disponibilidad real para todos los habitantes del planeta, 
debido a que esta se distribuye de manera geográfica y no de forma administrativa, es decir, su disponibilidad depende de la altitud, latitud, clima, entre otros factores ambientales, no de la manera en que los habitantes seleccionan los espacios territoriales para formar sus comunidades influenciadas por intereses políticos y económicos (Toledo, 2002).

La relación de los humanos con los recursos ambientales como el agua siempre ha sido una cuestión de política, dadas las formas de organización para explotar los medios a su alcance para cubrir sus necesidades y para resistir los embates que de esta emanan (como la escasez y el encarecimiento) (Lipietz, 1999, p. 204).

A partir de los años setenta, los recursos naturales y los problemas generados en torno a ellos comenzaron a ser materia de amplia discusión en las universidades, en los círculos científicos y entre los organismos internacionales, cuyos argumentos y aportaciones sobre las mejores prácticas de aprovechamiento y utilidad quedaron plasmados en iniciativas como la Carta de Estrasburgo, en 1968, la Declaración de Estocolmo, en 1972, y el Plan de Acción de Mar del Plata, en 1977.

Desde el ámbito académico-científico emergieron diferentes posicionamientos teóricos como la corriente de la eficiencia económica, que apoyada del análisis costo-beneficio argumentó que el mercado debía fungir como regulador de la explotación de los recursos naturales y del ambiente para buscar su existencia en el tiempo (Corona, 2000). Con estos precedentes, a partir de los años noventa fue más que evidente el uso creciente de recursos como el agua dulce para consumo humano y sus efectos irreversibles, al tiempo que se reconoció su valor social y económico (Tortajada, 2011).

En el caso de México, la Ley de Aguas Nacionales de 1994 eliminó el orden de prelación del agua, dejando que dicho recurso se rigiera bajos las condiciones de oferta y 
la demanda del mercado. A partir de esta condición mercantil, el agua dejó de considerarse un bien natural para convertirse en un recurso de mercado, cuyo valor de uso, de existencia y de herencia, aunado a sus atributos, permitió crear un mercado hipotético: de acuerdo al método de los precios hedónicos, su valor se determina con base en sus propias características y al valor interno del producto.

Incluso, la economía ambiental asume que los recursos, aun y cuando son naturales, independientemente de su abundancia o escasez deben ser valorados monetariamente dentro del mercado a través de la interacción directa entre los interesados (negociación de mercado) o mediante la intervención pública (aplicación de una carga fiscal por uso o desaprovechamiento). En esta misma línea versó la postura del economista neoclásico León Walras, al asumir que todo recurso natural (como el agua) puede concebirse como bien económico si su existencia es limitada con carácter apropiable, máxime si genera utilidad y bienestar individual y colectivo (Corona, 2000).

Es claro que la valoración monetaria del agua, concebida ahora como capital privado, es motivo de conflicto entre partes, ya que su denominación hedónica puede convertirse en un problema ético pues su pecunia no siempre refleja el valor de uso y su carácter no renovable. Más bien, lo que determina esta asignación es el costo de extracción, tratamiento, distribución y disposición, mismo que varía acorde a las características orográficas del territorio. Esta valoración monetaria, en términos de la administración pública se traduce en tarifas o pagos por concepto de uso o disposición de dicho recurso, convertido ahora en servicio; sin embargo, generalmente no están incluidos los costos ambientales, de aquí que al establecer las tarifas no sólo se deben consideran los aspectos técnicos (que por sí solos 
tendrían dificultades para definir el costo real del agua), sino también los aspectos sociales y políticos (FEA, et al., 2006). ${ }^{1}$

En afán de definir un valor nominal al agua dulce, algunos países ya han implementado diversos métodos e instrumentos no sólo para racionalizar su uso, sino también para ampliar su cobertura ante la creciente dinámica demográfica y el aumento de la demanda de dicho recurso. A sabiendas de que este no es renovable, su calidad frecuentemente indeseable (por la contaminación) y su disponibilidad cada vez más limitada han provocado que el precio se incremente gradualmente, tal como se aprecia en el Cuadro 1, lo que se convierte en una seria restricción a su consumo, al grado de considerarla como un recurso club ("club godos") cuyo disfrute será para quien pueda pagar o asumir el costo correspondiente (Zegarra, 2014).

Utilizando los preceptos malthusianos de la teoría económica clásica, se asume que la valoración nominal creciente del agua para consumo humano es hasta cierto punto justificable en razón de su carácter no renovable, por lo que el mercado debe ser capaz de asignarle un precio para racionalizar su uso y así evitar rendimientos productivos decrecientes y efectos en el nivel de bienestar individual y colectivo.

En este tenor, la teoría del desarrollo humano contraargumenta que los bienes y satisfactores básicos como el agua no debe tener un valor muy elevado para garantizar su consumo y evitar la degradación creciente de la calidad de

I. Cabe diferenciar entre tarifa y precio (cuota) del agua. La primera se refiere a los cargos monetarios que debe cubrir el usuario frente a una institución pública o concesionario al recibir dicho servicio, independientemente de la distancia o ubicación del demandante; el monto que paga es constante dada la existencia de una base normativa (precio administrado). El precio, por otro lado, además de partir sobre preceptos normativos, considera la disponibilidad, distancia, calidad del agua, presión y número de demandantes para asignar el valor final a dicho recurso, es decir, en esta definición nominal intervienen las condiciones del mercado (precio asociado) (Zegarra, 20l4). 
vida. Ello implica que los precios que se le asignen al agua, como recurso básico de sobrevivencia humana, deben ser coherentes con la capacidad adquisitiva de cada segmento social, de tal manera que se vele por la existencia del recurso y el bienestar social.

\section{Cuadro 1. Precio del agua en algunos países del mundo}

(2005-2014)

Precios por $m^{3}$ de agua para consumo humano

País

Canadá

EUA

España

Irlanda

Suecia

Italia

Finlandia

Holanda

Reino Unido

Francia

Bélgica

Alemania
2005

0.31

0.60

0.47

0.61

0.69

0.70

0.77

1.30

1.28

1.35

1.55

2.16 (en dólares corrientes)

2010

1.55

3.00

2.30

3.00

3.40

3.50

3.80

6.50

6.40

6.70

7.80

10.80
2014

1.55

3.10

2.30

3.00

3.50

3.50

3.90

6.50

6.50

6.80

7.70

10.80

Fuente: elaboración propia con base en datos históricos obtenidos de FEA, et al. (2006).

Implicaciones multidimensionales de la disponibilidad y calidad de agua para consumo humano

Cualquier sistema que pretende la gestión sostenible del territorio debería prescindir de la vulgar acepción de urbanizar, puesto que esta es una actividad que causa desmedido impacto ambiental al consistir básicamente en eliminar suelo rústico y todos sus componentes mediante un desproporcionado consumo de recursos escasos. En este 
sentido, los estragos que genera el avance de la urbanización son vastos, siendo tal vez uno de los más significativos el agotamiento del agua, que como elemento vital paradójicamente puede ser portadora de sufrimiento y muerte.

De acuerdo con la Organización Mundial de la Salud (OMS), al menos cinco millones de personas (en su mayoría niños menores de cinco años) fallecen cada año a causa de enfermedades transmitidas por el agua, situación que podría prevenirse con mejoras sanitarias y mejor suministro de agua potable. Esta situación da como resultado una mortalidad promedio de 13700 personas al día (Miller, 1992).

A principios del año 2000, el 70\% de la población mundial no contaba con agua salubre en cantidades adecuadas. El 90\% de la población rural del tercer mundo utilizaba habitualmente este tipo de agua, con sistemas de desagües domésticos situados dentro de sus casas o cerca de ellas, contaminando el suelo, los alimentos y el agua misma. Actualmente, en algunas partes del mundo, el agua no sólo está contaminada, sino que está escaseando, por lo que su calidad $^{2}$ disminuye y se degrada. Los mantos freáticos que se utilizan como fuente de agua potable están contaminados de plaguicidas, fertilizantes y sustancias químicas peligrosas.

En su paso a través del ciclo hidrológico, el agua se contamina principalmente por:

- Agentes microbiológicos, proveniente de desechos de aguas municipales no tratadas.

- Sustancias químicas de desechos industriales.

- Fertilizantes y plaguicidas.

- Intrusión salina.

- El sedimento que se deslava de la tierra y llega a las aguas superficiales por erosión natural y por la erosión acelerada del suelo a causa de la agricultura, silvicul-

2. La calidad del agua es la condición que permite su uso para consumo humano: libre de microorganismos, sustancias químicas o sustancias radioactivas, además con olor, color y sabor aceptables. 
tura, minería, construcción y otras actividades deforestadoras y causantes de pérdidas de recursos naturales.

- Los desechos orgánicos procedentes de excreciones animales y humanas y las partes descartadas de material verde cortado o podado (FEA, et al., 2006; Monforte y Cantú, 2009).

Este cúmulo de contaminantes se filtra y degrada la calidad del agua, pues al momento de convertirse en potable ya tiene una serie de impurezas suspendidas como bacterias, algas y protozoarios, que le dan olor, color y turbidez, y fango, que provoca opacidad, entre otros, mientras que las impurezas disueltas son sales (calcio y manganeso, bicarbonato, carbonato, sulfato y cloruro) que le dan alcalinidad, dureza y corrosión al agua, sodio (fluoruro) que actúa sobre el esmalte de los dientes, óxido de hierro, colorantes vegetales que le dan color y acidez al líquido, y gases (oxígeno, anhídrido carbónico, sulfahídríco y nitrógeno).

$\mathrm{Al}$ ser consumida con esta calidad, el agua puede generar severos efectos sobre la salud humana, siendo los más frecuentes tifos, gastroenteritis, esquistosomiasis, paratifos, hepatitis infecciosa, cólera asiática, disentería amebiana y bacilar, ántrax, tolerámica, tuberculosis, entre otros nuevos riesgos humanos que van surgiendo. En 1998, se estimó que hubo 2200000 muertos a causa de enfermedades diarreicas; entre ellos, más de 1800000 fueron menores de cinco años. Las heces humanas fueron el contaminante que afectó más gravemente la salud de los niños y niñas, aunque no constituyó la única amenaza, ya que también contaminaron el agua otras sustancias letales como el arsénico, el fluoruro y los nitratos radiactivos, todo lo cual representó una seria amenaza a la salud de las personas (Toledo, 2002).

En el año 2000, sólo seis países en el mundo disponían del $40 \%$ del total del agua del planeta y otros, a pesar de contar con el recurso, lo recibían estacionalmente, es decir, 
no disponían de este en todas las épocas del año, salvo por su almacenamiento en las presas o por las extracciones del subsuelo. Por lo tanto, cerca del 75\% de la población disponía sólo del $20 \%$ del total del agua, y se estima que para el año 2025 el 80\% de los habitantes del planeta vivirán bajo condiciones de alta y muy alta escasez de agua dulce apta para consumo humano (Toledo, 2002).

Para el año 2005, más de ochenta países que albergaban a $40 \%$ de la población mundial sufrieron grave escasez de agua, y el número de personas sin servicios adecuados y saneamiento podría llegar a 4500 millones en los próximos veinte años; las poblaciones urbanas pobres serían las más vulnerables. Las condiciones tenderán a empeorar en los siguientes cincuenta años, en la medida que aumente la población y el cambio climático global perturbe los regímenes de precipitaciones (FEA, et al., 2006).

En el Cuadro 2, se aprecia este panorama, donde Europa Occidental, Asia Central y del Sur y África del Norte y del Sur son las regiones que más problemas hídricos de consumo humano presentan, pues la cantidad de agua dulce en sus subsuelos es muy inferior a la tasa de crecimiento y demanda poblacional, lo que explica, en parte, los altos niveles de marginación y pobreza que caracterizan a dichas regiones. El resto de los países no escapa de esta situación racionada del agua, ya que el nivel de reposición (regularmente por lluvia) es cada vez más lento y la demanda per cápita del agua dulce es creciente, lo que deja en claro la disputa creciente de este recurso, haciéndolo cada vez más escaso y altamente valorado. 
Cuadro 2. Disponibilidad regional de agua para consumo humano (2005-2014)

Región

Oceanía

Sudamérica

África Central

América del Norte

Europa del Este

Europa Occidental

Asia Central y del Sur

África del Sur

África del Norte
Metros cúbicos anuales (promedio per cápita)

$\begin{array}{rrr}2005 & 2010 & 2014 \\ 53,715 & 53,875 & 54,033 \\ 36,990 & 37,100 & 37,250 \\ 20,890 & 20,952 & 21,014 \\ 16,805 & 16,852 & 16,902 \\ 14,820 & 14,864 & 14,907 \\ 1,773 & 1,777 & 1,782 \\ 1,467 & 1,471 & 1,474 \\ 1,291 & 1,294 & 1,297 \\ 497 & 498 & 498\end{array}$

Fuente: elaboración propia con base en datos históricos obtenidos de FEA, et al. (2006).

En el Cuadro 3, es perceptible la existencia de países latinoamericanos como Ecuador, El Salvador, Nicaragua, entre otros, donde la mayor parte de la población no sólo carece de dicho recurso, sino que también no lo dispone en condiciones adecuadas, es decir, el agua en dichos países no está entubada o no esta tratada apropiadamente para que sea consumida directamente en el domicilio, haciendo que la carestía sea todavía mucho más compleja y delicada, pues a medida que la población y la demanda aumentan, la oferta se merma cada vez más.

En los años posteriores a 2005 se presentó una mejora en la cobertura del agua, sin embargo, esta fue marginal, por lo que no deja de ser preocupante para aquel segmento que todavía no tiene acceso a dicho recurso, lo cual no sólo mina la capacidad de desarrollo del ser humano en sociedad, sino que también entorpece la dinámica económica de los propios países al ir desencadenando otros problemas como manifestaciones y revueltas sociales, conflictos y enfrentamientos gubernamentales, mayor gasto público, perforación y sobreexplotación de pozos hídricos artesanales, comercia- 
lización ilegal del agua, entre muchos otros más que al final afectan la estabilidad de los mercados internos.

Cuadro 3. Situación del agua para consumo humano en algunos países latinoamericanos (2005-2014)

País $\%$ de población con agua para consumo humano

\begin{tabular}{lccc} 
& 2005 & 2010 & 2014 \\
Costa Rica & 99 & 98 & 90 \\
Chile & 92 & 93 & 92 \\
Jamaica & 86 & 87 & 87 \\
Belice & 85 & 85 & 84 \\
Colombia & 76 & 76 & 75 \\
Rep. Dominicana & 73 & 74 & 73 \\
Bolivia & 72 & 73 & 72 \\
Brasil & 71 & 73 & 70 \\
Guatemala & 67 & 68 & 67 \\
Perú & 67 & 68 & 66 \\
Nicaragua & 63 & 64 & 62 \\
Ecuador & 56 & 57 & 56 \\
El Salvador & 53 & 54 & 53 \\
\hline
\end{tabular}

Fuente: elaboración propia con base en datos históricos obtenidos de FEA, et al. (2006).

Este ambiente refleja que la distribución del agua dulce es desigual entre las regiones naturales y económicas del planeta. Adicionalmente, el crecimiento sin planeación trae consigo la desigualdad social debido a que las regiones con mayores recursos económicos tienen más acceso al agua, independientemente de los costos económicos, sociales o ambientales. Por otra parte, además de la distribución asimétrica de este recurso, la situación de escasez se ve agravada por los niveles de consumo cada vez más influenciados por los estilos de vida asociados al proceso de urbanización (Monforte y Cantú, 2009). 
En el caso de México, la restricción a este recurso no deja de ser preocupante, razón por la que todavía el 36\% de la población nacional depende de los pozos artesanales como principal fuente de abastecimiento hídrico. En entidades como Yucatán, el suministro por este medio es del 80\%, mientras que en Quintana Roo es del 77\%, en Campeche del 74\%, en Tabasco del 65\%, en Sinaloa del 52\%, en México del $50 \%$ y en Zacatecas del $49 \%$. Esta forma de aprovisionamiento constituye una solución a la carencia de dicho recurso, pero también genera un grave problema, ya que su acelerada explotación provoca desecación de los mantos freáticos, sobre todo en las zonas donde las precipitaciones pluviales o filtraciones son muy irregulares, traduciéndose en una descompensación de la capacidad de recarga, pérdida o reducción de cubierta vegetal y erosión del suelo, afectando a su vez el desempeño de las actividades agrícolas y ganaderas principalmente.

Son seiscientos cincuenta los acuíferos distribuidos en el país que suministran aproximadamente la tercera parte de la extracción nacional para todos los usos; $15 \%$ está seriamente sobreexplotado y la mayoría de ellos están contaminados, situación que ha creado problemas de intrusión salina en dieciocho acuíferos situados en cinco estados costeros (FEA, et al., 2006).

En 1991, las viviendas con agua entubada o de acequia alcanzaban el 41\%, de las cuales únicamente el 17\% recibía el líquido en su interior. Si bien para el último trimestre de 2014 este porcentaje alcanzó el 85\%, sigue estando en desventaja el segmento de población carente. Por ejemplo, en la Ciudad de México para 1991 sólo el 46\% tenían agua al interior y $47 \%$ agua en el exterior de la vivienda, en tanto los porcentajes en Colima fueron del 31\% y $30 \%$, en Baja California Norte del 29\% y 26\%, y en Nuevo León del 27\% y $23 \%$ respectivamente (Barragán, 1994). 
Evidentemente, es en las zonas urbanas donde se cuenta con mayores porcentajes de viviendas con servicio de agua potable, pero también donde la lucha por la apropiación del recurso es más intensa. Para la capital del país, el problema del agua involucra tanto el encontrar las fuentes de abastecimiento como su distribución y posterior recolección en forma de aguas residuales. Es claro que el acelerado proceso de urbanización como el que se vive en la región centro del país requiere de una demanda creciente de este recurso, lo que obliga a localizar fuentes de abastecimiento como Valle de Bravo, Villa Victoria, Temascaltepec y Atlacomulco para abastecerse, incluso a costa del perjuicio de la sociedad de estos lugares. Tal es el caso de las localidades rurales de los municipios de Valle de Bravo y Villa Victoria, donde alrededor de una tercera parte de las viviendas carecen de este satisfactor básico (INEGI, 2010; Barragán, 1994).

El intento por resolver tal problema no es equitativo, es decir, las autoridades tratan en mayor medida de abastecer primero a las clases sociales mejor posicionadas económicamente dentro de los núcleos centrales, mientras que los segmentos de las zonas rurales y marginadas sufren los estragos de la falta de este líquido. Este hecho genera anarquía, polarización y heterogeneidad en la disponibilidad de tal recurso. A ello se suma la existencia de infraestructura para la distribución hidráulica, que no siempre presenta las mejores condiciones y cuyo deterioro contribuye a la reducción de la presión y el volumen hídrico, esto último derivado de desaprovechamientos por fugas o por descuidos humanos.

Se estima que la cantidad mínima que requiere un habitante para satisfacer sus necesidades de sed, aseo personal y servicios en las zonas urbanas es de 250 litros/habitante/día (l/h/d) en promedio, sin embargo, el deterioro de las redes de distribución hidráulica contribuye a que la disposición de dicho volumen sea menor, pues se pierden alrededor de 
$100 \mathrm{l} / \mathrm{h} / \mathrm{d}$ por fugas de diferente tipo y origen. Tanto la cantidad mínima requerida como la pérdida por fugas varían en función del clima de la región donde se habita, el nivel socioeconómico de la persona, sus estilos y formas de vida, la disponibilidad de agua en la zona y la accesibilidad a dicho recurso, la actividad ocupacional, el nivel de cultura de las personas, entre muchos otros factores más. Por ejemplo, en Tijuana, Baja California, el requerimiento es de $176 \mathrm{l} / \mathrm{h} / \mathrm{d}$; en León, Guanajuato, de 116; en Monterrey, Nuevo León, de 180; en Mexicali, Baja California, de 220; y en Naucalpan, Estado de México, de 225 litros/habitante/día (INEGI, 2010).

En el medio rural, donde los estilos de vida son menos dinámicos y donde en muchas ocasiones se cuenta con sistemas formales de abasto de agua, el requerimiento promedio es de $150 \mathrm{l} / \mathrm{h} / \mathrm{d}$, pero, al igual que en la zona urbana, las condiciones de la infraestructura generan problemas de desaprovechamiento, ocasionando pérdidas de $50 \mathrm{l} / \mathrm{h} / \mathrm{d}$ (FEA, et al., 2006).

Las condiciones de la disponibilidad de agua para consumo humano en el Estado de México

En lo contemporáneo, la disputa por el acceso al agua se ha vuelto un problema muy complejo y tema recurrente de la política pública, explicada en parte por el crecimiento de la población, de las viviendas y de la propia demanda de dicho bien. En el Estado de México, el número de viviendas pasó de 2218702 a 2668118 entre el año 1995 y el 2000, es decir, hubo un incremento de poco más de 400000 nuevos espacios humanos habitables, mientras que la población pasó de 11701934 a 13083359 habitantes, equivalente a una tasa de crecimiento de $11.78 \%$ en este mismo periodo, lo que significó la existencia de 1378425 habitantes más (INEGI, 2010). 
En el Cuadro 4, se aprecia que en los siguientes catorce años se presentó un tasa de crecimiento acumulado de $61.6 \%$ en el número de viviendas, para llegar a 4311929 en el último trimestre de 2014. En este mismo lapso, la población creció 25.6\% (INEGI, 2000, 2005 y 2010). Al contrastar esta dinámica creciente con la disponibilidad de agua para consumo humano se abre una gran brecha, convirtiéndose esta en un recurso que cada vez es más escaso, caro y de difícil acceso.

Cuadro 4. Crecimiento de las viviendas y la población total en el Estado de México (2005-2014)

Variable

\begin{tabular}{rrr}
2005 & \multicolumn{1}{c}{2010} & \multicolumn{1}{c}{2014} \\
3244378 & 3749499 & 4311929 \\
14007495 & 15175862 & 16435458 \\
\hline
\end{tabular}

Número de viviendas

en datos históricos de INEGI (2010).

Fuente: elaboración propia con base en datos históricos de INEGI (2010).

De 1996 a 1999, el Estado de México tuvo un déficit de $6 \mathrm{~m}^{3} / \mathrm{s}$, resultado de una demanda de $38 \mathrm{~m}^{3} / \mathrm{s}$ cuando el caudal disponible era de sólo $32 \mathrm{~m}^{3} / \mathrm{s}$. En afán de cubrir esta demanda, se generó una sobreexplotación del recurso, ya que se extrajeron 1619 millones de $\mathrm{m}^{3}$ de los mantos acuíferos del Valle de México, cuando la recarga era de únicamente 788.4 millones de $\mathrm{m}^{3}$; se trataba, pues, de una capacidad de reposición menor al 50\% (INEGI, 2000).

En este mismo sentido, el déficit hídrico en los diecisiete municipios mexiquenses más poblados ${ }^{3}$ de los veintisiete que colindan con la Ciudad de México fue de $3.5 \mathrm{~m}^{3} / \mathrm{s}$ de los $22.8 \mathrm{~m}^{3} / \mathrm{s}$ que demanda en promedio la misma población (Camacho, 1998). Esta carencia fue mucho más aguda en las zonas habitacionales de interés social de reciente creación

3. Ecatepec de Morelos, Nezahualcóyotl, Naucalpan de Juárez, Toluca, Tlalnepantla, Chimalhuacán, Tultitlán, Cuautitlán Izcalli, Atizapán de Zaragoza, Ixtapaluca, Nicolás Romero, Tecámac, Valle de Chalco Solidaridad, Chalco, Coacalco de Berriozabal, La Paz y Huixquilucan. 
y en zonas rurales y periféricas urbanas. Datos oportunos del año 2000 evidenciaron que la disponibilidad de este vital líquido se tornó cada vez más difícil y compleja, pues se siguió presentando un déficit de $2 \mathrm{~m}^{3} / \mathrm{s}$ en el Estado de México y en la zona metropolitana del Valle de México (ZMVM), cuya tendencia seguirá siendo de carestía, tal como se aprecia en el Cuadro 5.

Incluso, la diferencia entre la dotación deseable de agua potable (que debe ser de al menos de 250 litros per cápita promedio por día) y la dotación real es muy grande, es decir, los hogares de la entidad mexiquense carecen de poco más de 100 litros por habitante diariamente para desarrollar sus actividades, siendo más drástica esta limitación en los municipios mexiquenses colindantes con la Ciudad de México.

\section{Cuadro 5. Disponibilidad de agua de consumo humano en el Estado de México (2005-2014)}

Región

Estado de México

Cuenca del Valle de México-Pánuco

Municipios conurbanos de la Ciudad de México

Estado de México

Cuenca del Valle de México-Pánuco

Municipios conurbanos de la Ciudad de México
Demanda de agua potable $\left(\mathrm{m}^{3} / \mathrm{s}\right)$ $2005 \quad 2010 \quad 2014$

$45 \quad 51 \quad 53$

$\begin{array}{lll}37 & 41 & 43\end{array}$

$\begin{array}{lll}34 & 37 & 39\end{array}$

Oferta de agua potable $\left(\mathrm{m}^{3} / \mathrm{s}\right)$

$\begin{array}{lll}44 & 49 & 51 \\ 34 & 39 & 41 \\ 30 & 36 & 36\end{array}$

Fuente: elaboración propia con base en INEGI (2000 y 2010).

Algunas evidencias reflejan que:

- En Tlalnepantla, fue reducido el suministro de agua potable por la Comisión del Agua del Estado de México (CAEM, antes Comisión Estatal de Agua y Saneamiento 
-CEAS-) en 300 l/s diarios, lo que afectó al 70\% de la población.

- En la zona norte de Nezahualcóyotl, se carecía del suministro de agua desde principios de febrero de 1998; hoy se ha regularizado, pero todavía existen muchos problemas y carencias.

- En Metepec, se redujo el consumo del agua potable (zonas populares) y se incrementaron las tarifas hasta en un $50 \%$.

- Los otomíes de Santa María Tixmadeje y de la comunidad de Doxteje (municipio de Atlacomulco) recorren hasta tres kilómetros para obtener agua, dada la carencia de dicho recurso en o cerca de sus viviendas (Milenio, 2014).

Aunado a esta problemática está el valor nominal que se le ha dado al recurso. Por ejemplo, en el último trimestre del año 2014, el metro cúbico de agua en bloque ${ }^{4}$ que recibieron los municipios mexiquenses a través de la CAEM de acuerdo al tipo de localidad tuvo un costo promedio de MXN 7.00. Cabe mencionar que dicho valor se definió en función de la cantidad de demanda de agua, la distancia del lugar de extracción al punto de disposición final, el tipo de actividad que prevalece en el lugar y el ingreso per cápita promedio (CFEMYM, 2014).

Con base en esto, la entidad mexiquense se dividió en seis grandes grupos, tal como se aprecia en la Imagen 1. El primero estuvo integrado por los municipios conurbados de la Ciudad de México: Atizapán de Zaragoza, Coacalco, Cuautitlán, Cuautitlán Izcalli, Ecatepec, Naucalpan, Tlal-

4. De acuerdo al Código Financiero del Estado de México y Municipios, el agua en bloque consiste en la conexión o suministro de dicho recurso a un grupo de municipios o localidades bajo las mismas condiciones en términos de tiempo, presión, calidad y costo. 
nepantla y Tultitlán, cuyo valor del agua en bloque fue de MXN 8.04 por metro cúbico.

En el segundo grupo, con un costo de agua en bloque de MXN 7.43, se agruparon los municipios de Chimalhuacán, Huixquilucan, Ixtapaluca, La Paz, Lerma, Nezahualcóyotl, Nicolás Romero, Tepotzotlán, Toluca y Valle de Chalco Solidaridad. Adicionalmente, los usuarios que tuvieran asignación directa de la Comisión Nacional del Agua (CONAGUA) y utilizaran la infraestructura de la CAEM pagaron por cada metro cúbico asignado MXN 2.27 adicionales, y por el suministro en bloque, mediante la venta de cupones y entrega de carros tanque, erogaron a los Ayuntamientos MXN 11.4 por $\mathrm{m}^{3}$.

Imagen 1. Costo del agua en bloque para consumo humano en el Estado de México, 2014
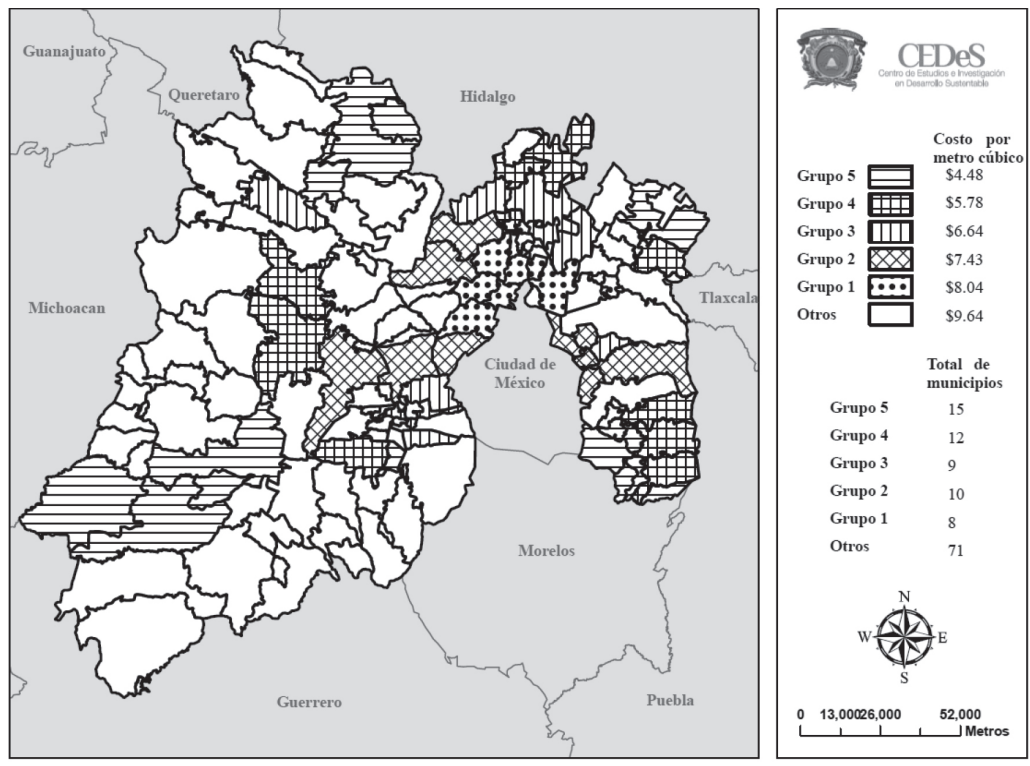

Fuente: elaboración propia con base en CFEMYM (2014). 
En el tercer grupo, con valor del agua en bloque de MXN 6.64, se consideraron los municipios de Atlacomulco, Chicoloapan, Huehuetoca, Nextlalpan, Ocoyoacac, Tecámac, Santiago Tianguistenco, Tultepec y Zumpango. En el cuarto grupo, con agua en bloque con valor de MXN 5.78, quedaron Almoloya de Juárez, Amecameca, Atlautla, Coyotepec, Hueypoxtla, Ixtlahuaca, Jaltenco, Otumba, Tenango del Valle, Teoloyucan, Tequixquiac y Tlalmanalco.

Axapusco, Ayapango, Ecatzingo, Jilotepec, Joquicingo, Juchitepec, Luvianos, Ozumba, San Simón de Guerrero, Soyaniquilpan de Juárez, Tejupilco, Temascaltepec, Tenango del Aire, Tepetlixpa y Timilpan integraron el bloque cinco, cuyo costo de suministro fue de MXN 4.48 por $\mathrm{m}^{3}$. Los municipios restantes, más alejados al Valle de México, ubicados al norte y sureste de la entidad, cuyo carácter es eminentemente agrícola y rural, se agruparon en el bloque seis, asignándoles la cuota más alta: MXN 9.64 por $\mathrm{m}^{3}$. Este monto se determinó en función de la distancia entre el centro de extracción-distribución del agua y la ubicación de las zonas habitacionales y viviendas, cuya dispersión y traza irregular provoca incrementos en la inversión de infraestructura, así como mayor desaprovechamiento por fugas.

A pesar de este valor tan alto, dichos municipios pueden coordinarse y asociarse para solicitar una reducción de la cuota establecida partiendo de sus condiciones económicas o de su disponibilidad financiera. En el caso de que en estas demarcaciones existan localidades indígenas o colectivos de alta marginación, podrán coordinarse, asociarse o firmar algún convenio con la CAEM o el organismo regulador respectivo para que se les exente o, en su caso, se les fije la cuota más baja en los términos y para los efectos que prevenga la ley.

Aunado al racionamiento y el costo por el acceso al agua, el desaprovechamiento o desperdicio que este recurso sufre es otra de las grandes preocupaciones que, si bien no es un 
problema propio de la entidad mexiquense, debe recibir especial atención, pues se estima que se pierde $40 \%$ del agua de distribución por las fugas en las tuberías. Algunas de las principales causas son (FEA, et al., 2006):

1) Deficiencias en la operación de la infraestructura para la captación y distribución del agua.

Este problema está relacionado con el bajo presupuesto que el Gobierno destina para la adecuada gestión de este recurso, ya que sólo 1.1\% del presupuesto nacional se asigna al rubro de agua, aunado esto a las bajas tarifas pagadas por los usuarios que en promedio sólo cubren MXN 1.73 por cada metro cúbico, cuando deberían pagar MXN 5.00 como precio mínimo. Si a esto se le agregan los serios problemas financieros para rehabilitar, mantener y operar la infraestructura requerida para captar, distribuir y tratar el agua, el problema se torna más complejo y costoso.

2) Los malos hábitos de consumo en los usuarios. Este problema es mucho más frecuente entre los usuarios que tienen acceso al agua de forma regular y abundante, actuando erróneamente bajo la idea de que dicho recurso es inagotable, y se debe a las bajas tarifas, la poca importancia que otorgan al mantenimiento y al uso de instalaciones de bajo consumo para lograr una utilización racional del agua, así como la ignorancia o todos estos factores en conjunto.

Lamentablemente, la mayoría de los usuarios tiene una percepción distorsionada sobre la contaminación, la sobreexplotación y otros efectos ambientales ocasionados por el aprovechamiento incorrecto del agua potable.

3) La falta de cultura de reúso, separación y aprovechamiento de agua de lluvia.

Todavía muchos segmentos de la sociedad no están acostumbrados a considerar otras posibilidades para cubrir la 
carencia de agua. No hay comercialización de aditamentos del hogar que busquen el reúso del agua ni la captación de agua de lluvia. Lo anterior no significa que la tecnología no se haya desarrollado, más bien es el precio alto lo que limita su uso.

En este tenor, en 1997 las pérdidas de agua potable por fugas en las redes primaria y secundaria de la Ciudad de México se calcularon en $37 \%$ del caudal con el que se abastecía a la capital. Esto representó más de 12000 litros por segundo, equivalente a una merma anual cercana a los 400 millones de metros cúbicos de agua potable. Estos desperdicios por fuga se explican por el rebase del periodo de vida útil de las tuberías, así como por las fisuras y fracturas originadas por hundimientos diferenciales del terreno y relacionadas al proceso de instalación, así como a la mala calidad de algunos materiales o a las conexiones defectuosas de tomas clandestinas.

En el año 2005, si bien se redujeron en un 58.3\% estas fugas, no dejó de ser preocupante la parte desaprovechada, que se calculó en 5000 litros de agua por segundo, recibiéndose alrededor de 23500 reportes de fugas de agua en ese año (FEA, et al., 2006). Esta situación representó una externalidad monetaria negativa muy alta, ya que el costo promedio por metro cúbico de agua recuperada fue de MXN 530700 000, equivalente al 50\% de lo que costaba incrementar un metro cúbico por segundo de nuevos caudales de fuentes externas. Tomando como referencia estos datos, se estima que en el último trimestre del año 2014 en esta misma entidad se desperdiciaron alrededor de 4160 litros de agua por segundo, cantidad menor respecto a 2005 pero con un costo de recuperación cercano al doble de lo que implicó en aquel año.

La pérdida fugaz de agua potable es descontrolada y puede dejar a varias ciudades sin abastecimiento en menos 
de una década, pero lo más grave es que a medida que se escasea dicho recurso el costo de disposición tiende a ser cada vez más caro. En 1998, el costo promedio por metro cúbico de agua potable desaprovechada por fugas fue de MXN 2.00; dieciséis años después (cuarto trimestre del año 2014) este costo se disparó hasta MXN 32.25, es decir, tuvo una tasa de crecimiento del $1500 \%$, explicada por el rápido crecimiento demográfico, la demanda per cápita creciente, la dispersión de las nuevas viviendas, el surgimiento de nuevos asentamientos humanos en espacios irregulares, el crecimiento de los establecimiento comerciales, de servicios e industriales, así como la alteración de los ciclos climáticos, cuyos periodos largos de sequía retardan la recarga de los mantos acuíferos, entre muchos otros factores.

En 1998, se estimó que en los municipios mexiquenses se desaprovechaban $15 \mathrm{~m}^{3} / \mathrm{s}$ por fugas, lo que equivalía a MXN 91250 por $\mathrm{m}^{3} / \mathrm{s}$ diarios que se quedaban en el subsuelo. A pesar de que en los años siguientes la cantidad desperdiciada aumentó marginalmente (6.6\% de 1998 al último trimestre del 2014), el costo de recuperación se disparó a una tasa de 540\%, tal como se aprecia en el Cuadro 6 (CFEMYM, 2014; Milenio, 2014).

En el último trimestre del 2014, algunos de los municipios mexiquenses con una mayor cantidad de agua potable desaprovechada por fugas fueron Nezahualcóyotl, Chalco y Los Reyes La Paz, esto debido al mal estado de las instalaciones domiciliarias y de la línea de distribución principal, lo que provocó un desaprovechamiento colectivo del $40 \%$ de dicho recurso.

Por su parte, en el municipio de Toluca la oferta de agua no había representado un problema hasta 1997 debido a la existencia del acuífero en Lerma que abastecía a sus habitantes y que incluso podía exportar el agua hacia la Ciudad de México. Sin embargo, hoy el panorama ha cambiado: existe una gran variabilidad en la dotación y disponibilidad 
del recurso, ya que hasta 1998 se tenía un déficit de más de 27 millones de $\mathrm{m}^{3}$ anuales. Una de las alternativas que adoptó el Gobierno local fue la perforación de pozos y la intensificación extractiva, hecho que contribuyó a mitigar el problema, pero que también generó grandes desequilibrios ambientales, hundimientos y fracturas viales en algunas zonas del municipio (Romo y Ávila, 2012).

Cuadro 6. Costo por desaprovechamiento de agua para consumo humano en el Estado de México (1998-2014)

\begin{tabular}{ccc} 
Año & $\begin{array}{c}\text { Volumen } \\
\text { desaprovechado }\left(\mathrm{m}^{3} / \mathrm{s}\right)\end{array}$ & $\begin{array}{c}\text { Costo } p o r \mathrm{~m}^{3} / \mathrm{s} \text { de agua } \\
\text { desaprovechada (en pesos corrientes) }\end{array}$ \\
1998 & 15 & 91250 \\
2000 & 15 & 182500 \\
2005 & 16 & 182500 \\
2010 & 16 & 365000 \\
2014 & 16 & 547500 \\
\hline
\end{tabular}

Fuente: elaboración propia con base en Milenio (2014).

El temor al agotamiento del agua para consumo humano, dotada de un precio de mercado, desaparecería si se permitiera jugar sin ninguna intervención a los mecanismos del mercado, pues a medida que se incrementaran los precios se generaría un estímulo a racionalizar su consumo, lo que sería en favor de estrategias de exploración y de investigaciones tecnológicas relativas a materiales, infraestructura y mecanismos de construcción para su distribución, evitando o reduciendo de esta manera el desaprovechamiento por fugas, ya que si bien los costos que ello implica son asumidos por el Estado, los efectos negativos (carencia o disponibilidad limitada, de baja calidad y alto costo) permean en el consumidor, inhibiendo consecuentemente la satisfacción de sus necesidades mínimas básicas de sobrevivencia. Esta concepción ha dado origen a la teoría económica de la explotación óptima de recursos naturales, que determina 
una trayectoria eficiente del agotamiento de los recursos y permite proseguir con el crecimiento económico y social a través del disfrute de los recursos procedentes de la naturaleza como el agua (Corona, 2000).

\section{Conclusiones}

La transformación del agua de consumo humano de un bien público a un recurso nominal mercantilizado está relacionada con su disponibilidad limitada, calidad y demanda creciente; un recurso cuyas múltiples funciones lo convierten en elemento necesario para el funcionamiento de la economía, la reproducción y la calidad de vida de la humanidad. Sin embargo, debido a múltiples factores, el agua ha acrecentado su escasez, no sólo en el Estado de México, sino en todas las escalas territoriales, lo que podría ser la razón de la asignación de un valor nominal para ella, lo cual desde la perspectiva de la teoría económica se convierte en un mecanismo de control y regulación de explotación, pero también en una restricción de uso social, lo que atenta contra el derecho a su disfrute.

Con esto se asume que el problema del agua en términos de escasez y encarecimiento es definitivo y su demanda cada vez más creciente. En México, y en la propia entidad mexiquense, los conflictos por la disponibilidad y acceso al agua son más que evidentes, por lo que constituyen una de las grandes preocupaciones de la sociedad contemporánea. La política pública debe atender urgente y puntualmente dicha situación, a través de la definición de mecanismos pertinentes y coherentes para su manejo y distribución, así como prever estrategias institucionales para enfrentar necesidades de construcción, ampliación o mantenimiento de infraestructura cada vez mayores, buscando reducir e incluso evitar los desaprovechamientos por fugas, esto en afán de compensar gradualmente el déficit presente en la 
mayoría de los municipios del Estado de México, sobre todo, por el claro incremento de la demanda aunado a la gran incertidumbre en su oferta.

Las tendencias demográficas en México plantean grandes retos en relación con el suministro, la distribución y el tratamiento de aguas. Dentro de quince años, de mantenerse las tasas actuales de crecimiento demográfico (1.8\%), la población será de 141 millones de habitantes, sin embargo, la disponibilidad de agua por habitante tenderá a reducirse notoriamente en los próximos veinte años y se tornará muy crítica y preocupante, pues se estima que dentro de veinticinco años habrá 25 millones de mexicanos más.

En la entidad mexiquense, el panorama es similar, e incluso más crítico aún, pues con la alteración creciente de los ciclos climáticos, principalmente la temporada de lluvia y la prolongación de los periodos de sequía, la disponibilidad natural de agua per cápita será cada vez menor, lo que implica que el déficit presentado en 2014 se incrementará y, por lo tanto, la competencia y los conflictos sociales por el acceso al vital líquido serán más recurrentes y menos atendibles, principalmente en los municipios colindantes con la Ciudad de México, por los costos que ello implica, ampliando consecuentemente las necesidades humanas y las brechas socioeconómicas entre estados y municipios.

Anderson, E. (1993). Value in ethics and economics. EE. UU.: Bibliografía Harvard University Press.

Azqueta, D., et al. (2007). Introducción a la economía ambiental. España: Mc Graw Hill.

Barragán, J. I. (1994). 100 años de vivienda en México, historia de la vivienda en una óptica económica y social. México: URBIS Internacional.

Camacho, C. (1998). Tercer informe de gobierno. México: Gobierno del Estado de México. 
Bibliografía

CFEMYM (2014). Código Financiero del Estado de México y Municipios. México: Gobierno del Estado de México.

Corona, A. (2000). Economía ecológica, una metodología para la sustentabilidad. México: UNAM.

FEA, et al. (2006). El agua en México: lo que todas y todos debemos saber. México: Centro Mexicano de Derecho Ambiental (CEMDA), Fondo Educación Ambiental (FEA), Presencia Ciudadana.

Flores, R. (20|4). Los afluentes y los ríos. La construcción social del medio ambiente en la cuenca Lerma-Chapala. México: ITESO.

Gleick, P. (2002). The World's Water: The Biennial Report on Freshwater Resources 2002-2003. Washington: Island Press.

INEGI (2000). Censo de población y vivienda, resultados definitivos, 2000. México: INEGI.

- (2005). Conteo de población y vivienda, resultados definitivos, resultados preliminares, 2000. México: INEGI. (2010). Censo de población y vivienda, 2010. México: INEGI.

Lipietz, A. (1999). ¿Qué es la ecología política? La gran transformación del siglo XXI. Santiago de Chile: LOM-IEP. Macnaghten, P., y Urry, J. (1998). Naturalezas impugnadas. Londres: Sage.

Milenio (2014). "Se definen tarifas del agua en el Estado de México”. Milenio Diario, p. 12.

Miller, T. (1992). Ecología y medio ambiente. México: Iberoamérica.

Monforte, G., y Cantú, P. C. (2009). “Escenario del agua en México”. Culcyt/Recursos Hídricos, 6(30), 3 I-40.

Romo, A. M., y Ávila, K. (2012). “Programa de saneamiento y calidad del agua: experiencias del municipio de Toluca en la aplicación de la NOM-002-SEMARNAT-1996", en A. Alvarado (coord.), Experiencias en el tratamiento de 
aguas residuales domésticas en el Estado de México (pp. | Bibliografía I 17- 147). México: UAEM-FAPUR.

SEMARNAT-SER (1992). Conferencia internacional sobre el agua y el medio ambiente: el desarrollo en la perspectiva del siglo XXI. México: SEMARNAT, SER.

Toledo, A. (2002). "El agua en México y en el mundo". Gaceta ecológica. Instituto Nacional de Ecología, (64), 9-I8. Tortajada, C. (20I I). El agua y el medio ambiente en las conferencias mundiales de las Naciones Unidas. Zaragoza: Agenda 21.

Zegarra, E. (20I4). Economía del agua. Conceptos y aplicaciones para una mejor gestión. Lima: GRADE. 